\title{
IncRNA MALAT1 promotes cell proliferation and invasion by regulating the miR-101/EZH2 axis in oral squamous cell carcinoma
}

\author{
LEI XIAO ${ }^{1^{*}}$, WEIPING WANG ${ }^{2 *}$, JINGJING ZHAO $^{3}$, HONGYAN XU $^{4},{\text { SHAOZHEN } \text { LI }^{5} \text { and XIUHUA YANG }}^{6}$ \\ ${ }^{1}$ Department of Stomatology, The Affiliated Yantai Yuhuangding Hospital of Qingdao University; ${ }^{2}$ Department of Stomatology, \\ Yantaishan Hospital, Yantai, Shandong 264000; ${ }^{3}$ Department of Surgery, The People's Hospital of Zhangqiu Area, \\ Jinan, Shandong 250200; ${ }^{4}$ Department of Stomatology, Shaanxi Provincial People's Hospital, Xi'an, Shaanxi 710000; \\ Departments of ${ }^{5}$ Imaging and ${ }^{6}$ Pharmacy, The People's Hospital of Zhangqiu Area, Jinan, Shandong 250200, P.R. China
}

Received September 16, 2019; Accepted June 3, 2020

DOI: $10.3892 / \mathrm{ol} .2020 .12024$

\begin{abstract}
Oral squamous cell carcinoma (OSCC) is the most common malignant tumor of the oral cavity. Emerging evidence indicates that long non-coding (lnc)RNAs play a key role in the cellular processes of tumor cells, including glycolysis, growth and movement. Here, the purpose of this study was to explore the biological functions and potential mechanism of lncRNA metastasis associated lung adenocarcinoma transcript 1 (MALAT1) in OSCC. OSCC tissues and adjacent matched paraneoplastic normal tissues were collected from 20 OSCC patients. The expression of MALAT1 and miR-101 in OSCC tissues and cell lines (HSC3, SCC9, SCC15 and SCC25) were determined by real-time-polymerase chain reaction (qPCR). Caspase-3, xaspase-8 and EZH2 protein levels were determined by western blot analysis. MALAT1-mediated miRNAs were verified by bioinformatics analysis of StarBase and Luciferase reporter assay. Cell Counting Kit-8 (CCK-8) and Transwell assays were used for investigating MALAT1 effect on cell proliferation and invasion in the OSCC cells. qPCR analysis indicated that MALAT1 expression was obviously increased, and miR-101 was decreased in the OSCC tissues and cell lines. Functional studies revealed that overexpression of MALAT1 promoted OSCC cell proliferation and invasion. Further experiments revealed that miR-101 was a target of MALAT1 and that the miR-101 inhibitor abolished the effect of MALAT1 on OSCC cell proliferation and invasion. Enhancer of zeste 2 polycomb repressive complex 2
\end{abstract}

Correspondence to: Dr Hongyan Xu, Department of Stomatology, Shaanxi Provincial People's Hospital, No. 256 Youyi West Road, Xi'an, Shaanxi 710000, P.R. China

E-mail: weijialiao63603@163.com

${ }^{*}$ Contributed equally

Key words: long non-coding RNA, MALAT1, microRNA-101, EZH2, oral squamous cell carcinoma subunit (EZH2) acted as a downstream effecter of MALAT1 in the OSCC cells. Collectively, these findings revealed that upregulation of MALAT1 facilitated OSCC proliferation and invasion by targeting the miR-101/EZH2 axis.

\section{Introduction}

Oral squamous cell carcinoma (OSCC) is the eighth most common tumor worldwide, and accounts for $2.1 \%$ of deaths caused by all cancers $(1,2)$. Oral cancer consists of a group of neoplasms that affect regions of the oral cavity, pharyngeal regions and salivary glands (3). Although great advances have been made in the treatment of OSCC, including surgery, chemotherapy, radiotherapy and hormonotherapy, the 5-year survival rate of OSCC remains less than $50 \%$, mainly due to cancer metastasis to lymph nodes (4). Therefore, there is an urgently need to explore the molecular mechanisms underlying OSCC to improve therapeutic efficacy and the survival rate of OSCC patients.

Long non-coding RNAs (lncRNAs) are a class of non-coding RNAs more than 200 nucleotides in length. Increasing evidence suggests that lncRNAs are notable molecular markers involved in the regulation of gene expression and cancer progression $(5,6)$. Dysfunction of IncRNAs are closely related to the incidence of human diseases, including tumors, degenerative neurological diseases and other major diseases that seriously endanger human health $(7,8)$. Thus, currently lncRNAs are a 'hot spot' of tumor research. lncRNA metastasis-associated lung adenocarcinoma transcript 1 (MALAT1) was first discovered in non-small cell lung cancer (NSCLC), and is located on chromosome 11q13.1 which is highly conserved (9). MALAT1 was reported to be upregulate in colorectal cancer (CRC) and to promote cell proliferation, metastasis and invasion (10). However, the molecular mechanisms of MALAT1 in mediating the progression of OSCC have not been well investigated.

MicroRNAs (miRNAs/miRs) are a large family of short non-coding RNAs, consisting of 18-25 nucleotides, and have been found to negatively regulate target genes via binding to the 3'-UTR (3'-untranslated region) (11). miR-101 has been 
reported to be downregulated in OSCC cells and to act as a tumor suppressor in OSCC development (12). It is worth mentioning that miR-101 was suggested to be a potential target of MALAT by Starbase. However, the exact role of miR-101 in MALAT-mediated OSCC progression needs further investigation.

As previously elucidated, miRNAs are involved in tumor development by targeting their mRNAs. Enhancer of zeste 2 polycomb repressive complex 2 subunit (EZH2) has been verified as a direct target of miR-101 in various types of cancers, including CRC, laryngeal squamous cell carcinoma and retinoblastoma (13-15). However, whether EZH2 serves as the target of miR-101 in the modulation of OSCC progression has not yet been clarified.

\section{Materials and methods}

Tissue specimens. Twenty paired OSCC tissues and adjacent normal tissues were obtained from The Affiliated Yantai Yuhuangding Hospital of Qingdao University (Yantai, Shandong, China) between March 2015 and April 2018. All patients (age range, 35-75 years, mean 61.3 years; 16 male and 4 female) were not treated with radiotherapy or chemotherapy prior to surgery. Liquid nitrogen was used to flash-freeze all the tissue specimens, and then the collected tissues were stored at $-80^{\circ} \mathrm{C}$ for further use. All patients signed written informed consent. This study was approved by the Ethics Committee of the Affiliated Yantai Yuhuangding Hospital of Qingdao University (no. 20140506).

Cell culture. OSCC cell lines (HSC3, SCC9, SCC15 and SCC25) and primary normal human oral keratinocyte (NHOK) cells were purchased from the BeNa Culture Collection (BNCC, Shanghai, China). RPMI-1640 medium containing 10\% FBS (Gibco; Thermo Fisher Scientific, Inc.), $100 \mathrm{U} / \mathrm{ml}$ penicillin and $100 \mu \mathrm{g} / \mathrm{ml}$ streptomycin were used to culture OSCC cells and then cells were incubated at $37^{\circ} \mathrm{C}$ with $5 \% \mathrm{CO}_{2}$ atmosphere.

Cell transfection. Small interference RNA (siRNA), scrambled oligonucleotide (NC) vector, MALAT1 siRNA, MALAT1 vector, miR-101 mimic, miR-101 inhibitor, negative control (NC)-mimic, NC-inhibitor, EZH2 plasmid and NC-plasmid were designed and synthesized by GenePharma Co. They were transfected into SCC 9 cells by Lipofectamine 2000 reagent (Invitrogen; Thermo Fisher Scientific, Inc.) according to the manufacturer's instructions. Forty-eight hours after transfection, the efficiency was validated by quantitative polymerase chain reaction (qPCR). miRNA sequences were as follows: miR-101 mimic sense, 5'-UACAGUACUGUGAUAACUGAA-3' and antisense, 5'-CAGUUAUCACAGUACUGUAUU-3'; control-mimic sense, 5'-UUCUCCGAACGUGUCACGUTT-3' and antisense, 5'-ACGUGACACGUUCGGAGAATT-3'; miR-101 inhibitor, 5'-UUCAGUUAUCACAGUAUGUA-3'; and inhibitor-control, 5'-CAGUACUUUUGUGUAGUACAA-3'.

CCK- 8 assay. Cell Counting Kit-8 (CCK-8, Beyotime Institute of Biotechnology) was applied for testing SCC9 cell viability. SCC9 cells were seeded onto 96-well plates at $1 \times 10^{3}$ cells/well with RPMI-1640 medium for 24, 48, 72 and $96 \mathrm{~h}$ in an incubator under normal conditions $\left(37^{\circ} \mathrm{C}, 5 \% \mathrm{CO}_{2}\right)$. Then, $\mathrm{CCK}-8$ solution $(10 \mu \mathrm{l})$ was added for incubation for another $2 \mathrm{~h}$ at $37^{\circ} \mathrm{C}$. Absorbance of each well was detected using a microplate reader (Bio-Rad Laboratories, Inc.) at $450 \mathrm{~nm}$.

Transwell invasion assay. SCC9 cells were inoculated in a prepared Transwell chamber $(8-\mu \mathrm{m}$ pore size; Corning Inc.) with Matrigel (BD Biosciences), and the cell density of each group was adjusted with serum-free RPMI-1640 culture medium. Briefly, $5 \times 10^{4}$ SCC9 cells were added to the upper chamber and RPMI-1640 culture medium containing $20 \%$ FBS was added to the lower chamber. The cells on the upper chamber were removed with a cotton swab. The cells that migrated to the lower chamber were fixed with $4 \%$ paraformaldehyde and stained with $0.5 \%$ crystal violet (Sigma-Aldrich; Merck $\mathrm{KGaA}$ ) for $15 \mathrm{~min}$ at room temperature. The number of transmembrane cells from 5 random different fields was selected under a microscope (magnification, x100; SZ61; Olympus).

Dual luciferase activity assay. Through Starbase online software (http://starbase.sysu.edu.cn/index.php), we searched for the potential target miRNAs of MALAT1. The MIMAT number of miR-101-3p is MIMAT0000099 (miRBase). Next, through the TargetScan online software (http:/www. targetscan.org/vert_71/), we searched for the potential target genes of miR-101. MALAT1 and EZH2 sequences containing the wild-type (WT) or mutated (MuT)-type miR-101 binding sites were cloned into the pGL3 luciferase reporter vector (pGL3-empty; Promega Corp.), respectively. SCC9 cells were co-transfected with miR-101 mimic along with MALAT1-WT/-MuT reporter or EZH2-WT/-MuT reporter using Lipofectamine 2000 reagent (Invitrogen; Thermo Fisher Scientific, Inc.), respectively. After a 48-h transfection, luciferase activity was examined using the Dual Luciferase Reporter Assay system (Promega Corp.). The values were normalized to those obtained for pre-miR negative control transfection.

Real-time-polymerase chain reaction ( $q P C R)$. TRIzol reagent (Invitrogen; Thermo Fisher Scientific, Inc.) was applied for extracting total RNA from OSCC tissues and cells. Complementary DNA (cDNA) was synthesized using the PrimeScript RT reagent kit (Takara). Then, RNA was reverse transcribed using reverse transcription kit (Takara). RT-PCR was performed using the SYBR-Green RT-PCR kit (Bio-Rad Laboratories, Inc.). The expression levels were analyzed using the $2^{-\Delta \Delta \mathrm{Cq}}$ method (16). Glyceraldehyde-3-phosphate dehydrogenase (GAPDH) and U6 were used as the internal reference for MALAT1 and miR-101, respectively. The primers are shown in Table I.

Western blot analysis. Total proteins were extracted using RIPA lysis buffer $(25 \mathrm{mmol} / \mathrm{l}$ Tris- $\mathrm{HCl}+150 \mathrm{mmol} / \mathrm{l} \mathrm{NaCl}$ $+1 \%$ NP-40 + $1 \%$ sodium deoxycholate $+0.1 \%$ SDS, $\mathrm{pH} 7.6$; Beyotime Institute of Biotechnology) supplemented with $1 \%$ protease inhibitors (100X; Roche Diagnostics) and phenylmethanesulfonyl fluoride (PMSF, $100 \mathrm{mmol} / \mathrm{l}$; Sigma-Aldrich; Merck KGaA). The protein concentration was detected by BCA kit (Beyotime Institute of Biotechnology). Equal 
Table I. Primer sequences for real-time fluorescence quantification PCR.

\begin{tabular}{ll}
\hline Gene & \multicolumn{1}{c}{ Primer sequences } \\
\hline GAPDH & Forward primer: 5'-GCACCGTCAAGGCTGAGAAC-3' \\
U6 & Reverse primer: 5'-ATGGTGGTGAAGACGCCAGT-3' \\
lncRNA MALAT1 & Forward primer: 5'-CTCGCTTCGGCAGCACA-3' \\
miR-101-3p & Reverse primer: 5'-AACGCTTCACGAATTTGCGT-3' \\
& Forward primer: 5'-GGGTGTTTACGTAGACCAGAACC-3' \\
EZH2 & Reverse primer: 5'-CTTCCAAAAGCCTTCTGCCTTAG-3' \\
& Forward primer: 5'-CGGCGGATCTTTGATAGACT-3' \\
& Reverse primer: 5'-GGAGGTGCTGGATGGAGTTA-3' \\
& Forward primer: 5'-TTCATGCAACACCCAACACT-3' \\
& Reverse primer: 5'-GAGAGCAGCAGCAAACTCCT-3'
\end{tabular}

MALAT1, metastasis associated lung adenocarcinoma transcript 1; EZH2, Enhancer of zeste 2 polycomb repressive complex 2 subunit; GAPDH, glyceraldehyde-3-phosphate dehydrogenase; lncRNA, long non-coding RNA.

amounts $(20 \mu \mathrm{g})$ of extracted proteins were separated by $10 \%$ SDS-PAGE and then transferred to NC membranes. Then, the NC membranes were blocked with $5 \%$ non-fat milk for $1 \mathrm{~h}$ at room temperature, and subsequently cultured with the primary antibodies, rabbit monoclonal anti-EZH2 (dilution 1:1,000, ab191080, Abcam), rabbit monoclonal anti-caspase-3 (dilution 1:1,000, ab197202, Abcam) and rabbit monoclonal anti-caspase-8 (dilution 1:1,000, ab25901, Abcam). The membrane was washed with TBST, and incubated with horseradish peroxidase conjugated goat anti-rabbit secondary antibody (ab6721, Abcam) at room temperature for $1 \mathrm{~h}$. Finally, the enhanced chemiluminescence (ECL) was applied for detecting the immune complexes. GAPDH was used as the internal control. The bands were subjected to quantification using the Image J imaging processing program (National Institutes of Health, Bethesda, MD, USA).

Statistical analysis. All data are expressed as mean \pm standard deviation (SD). Each experiment was repeated at least three times. The Student's t-test, one-way analysis of variance (ANOVA) and Pearson product moment correlation coefficient were used for statistical analysis in GraphPad Prism 6.0 software (GraphPad Software, Inc.). Kaplan-Meier curves and log-rank test were performed to analyze the survival rate of OSCC patients with different levels of MALAT1. Spearman's correlation analysis was implemented to validate the correlation between the levels of MALAT1 and miR-101 in OSCC tissues. $\mathrm{P}<0.05$ was considered statistically significant.

\section{Results}

MALAT1 is upregulated in OSCC tissues and cell lines. qPCR was carried out to detect MALAT1 expression in OSCC tissues and cell lines. As shown in Fig. 1A, the expression of MALAT1 was significantly increased in the OSCC tissues compared to that noted in the normal tissues. Then, MALAT1 expression was detected in OSCC cell lines using qPCR. As shown in Fig. 1B, MALAT1 expression was significantly increased in all OSCC cell lines (HSC3, SCC9, SCC15 and
SCC25) in comparison with that in normal NHOK cells. OSCC patients were divided into two subgroups (low/high MALAT1 level) using the median level of MALAT1 as a cut-off value. Kaplan-Meier survival analysis displayed that the overall survival was shorter in OSCC patients with high expression of MALAT1 compared to that with low expression of MALAT1 (Fig. 1C). We subsequently chose SCC9 for further research because it exhibited the highest MALAT1 expression in the 6 cell lines.

MALAT1 promotes OSCC cell proliferation and invasion. To clarify the role of MALAT1 in proliferation and invasion, MALAT1-vector or MALAT1-siRNA was transfected into SCC9 cells. Expression of MALAT1 was determined by qPCR. The findings showed that MALAT1 was downregulated by MALAT1-siRNA, while upregulated by MALAT1-vector when compared with the relevant NC control (Fig. 2A). The viability and invasive ability of SCC9 cells were measured by CCK-8 and Transwell assays, respectively. As shown in Fig. 2B, MALAT1 overexpression promoted SCC9 cell viability, while MALAT1 knockdown inhibited the viability of SCC9 cells. The results of western blot analysis displayed that the levels of caspase- 3 and caspase- 8 were decreased by the MALAT1-vector, while increased by MALAT1-siRNA (Fig. 2C). Additionally, the findings of the Transwell assay displayed that the invasiveness of the SCC9 cells in the MALAT1-vector group was significantly increased, whereas this ability was decreased in the MALAT1-siRNA group (Fig. 2D). The above-mentioned data indicate that overexpression of MALAT1 promotes the proliferation and invasion of OSCC cells.

MALAT1 directly interacts with miR-101 in OSCC cells. The bioinformatics analysis Starbase online software (http://starbase.sysu.edu.cn/index.php) was first performed to detect the potential miRNAs regulated by MALAT1. It was identified that miR-101 was the most probable target miRNA of MALAT1; miR-101 shared complementary binding sites with the 3'-UTR of MALAT1 (Fig. 3A). Dual-luciferase assay was 

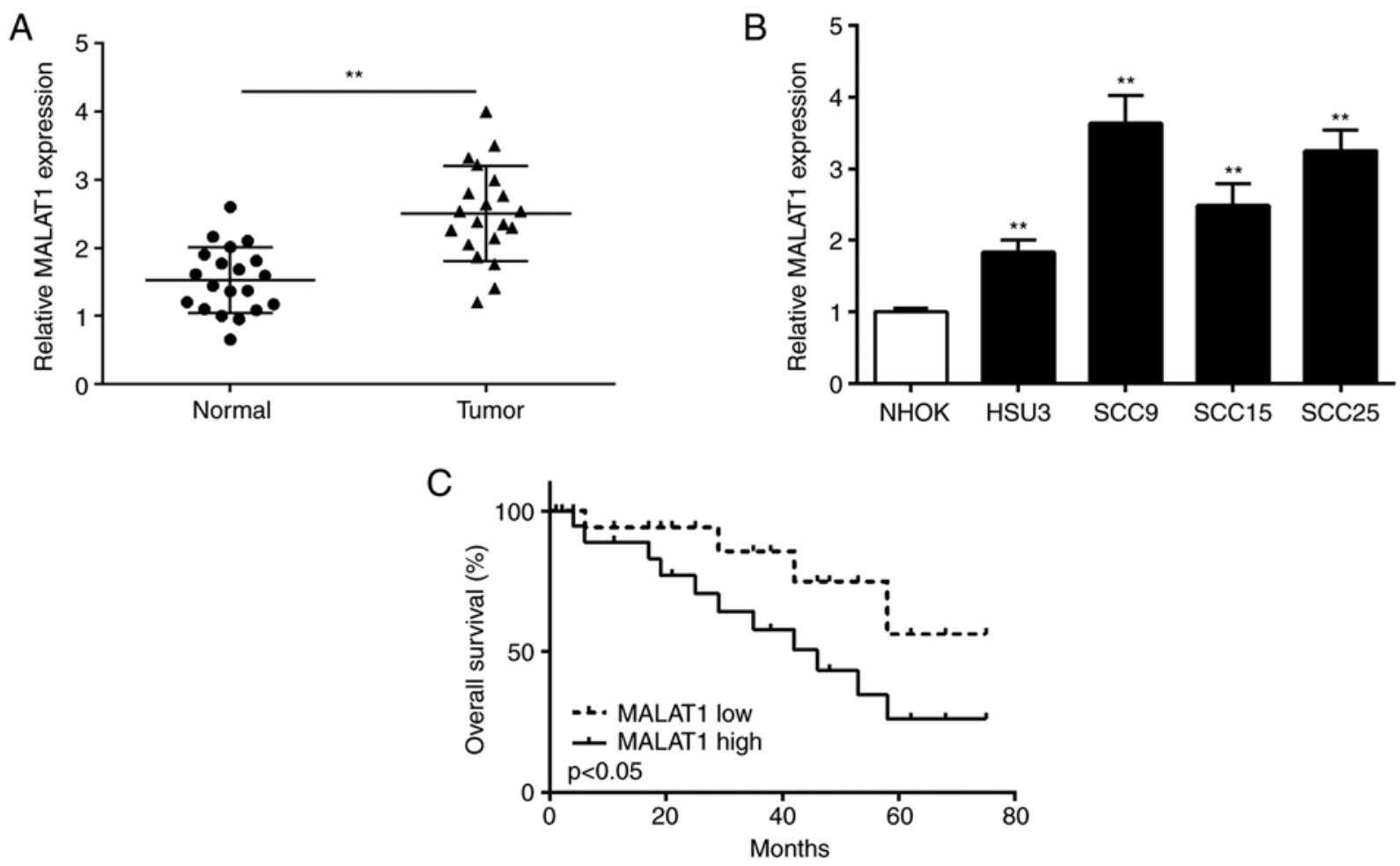

Figure 1. High expression of MALAT1 was verified in OSCC tissues and cell lines by qPCR. (A) MALAT1 expression was determined in OSCC tissues and paired normal tissues by qPCR $(n=20){ }^{* *} \mathrm{P}<0.01$. (B) MALAT1 expression was examined in OSCC cells and normal oral NHOK cells by $q \mathrm{PCR}$. ${ }^{* *} \mathrm{P}<0.01$, compared with NHOK cells. (C) Kaplan-Meier survival analysis of OSCC patients with high or low expression of MALAT1. OSCC, oral squamous cell carcinoma; MALAT1, metastasis associated lung adenocarcinoma transcript 1.

then performed to confirm the bioinformatical prediction. As shown in Fig. 3B, miR-101 mimic significantly downregulated the luciferase activity of MALAT1 3'-UTR-WT in SCC9 cells. However, there was no significantly change in the luciferase activity of MALAT1 3'UTR-MuT. Furthermore, the result of the qPCR analysis revealed that miR-101 was downregulated in the OSCC tissues when compared with the normal tissues (Fig. 3C). Notably, overexpression of MALAT1 inhibited miR-101 expression, while silencing of MALAT1 expression enhanced miR-101 expression in the SCC9 cells (Fig. 3D). More strikingly, a negative correlation was noted between MALAT1 expression and miR-101 in the OSCC tissues (Fig. 3E). Taken together, these results suggest that MALAT1 directly targets miR-101 in regulating OSCC progression.

miR-101 is associated with MALAT1-mediated OSCC cell proliferation and invasion. Next, we explored the biological effect of miR-101 as regulated by 1 ncRNA MALAT1 on OSCC cell proliferation and invasion. SCC9 cells were treated with the MALAT1-vector or MALAT1-siRNA, simultaneously with the miR-101 mimic. The results of the CCK-8 assay demonstrated that the viability of SCC 9 cells was increased by the MALAT1-vector yet significantly reduced by the miR-101 mimic (Fig. 4A). However, the viability of SCC9 cells was inhibited by MALAT1-siRNA which was further suppressed by miR-101 mimic (Fig. 4B). Moreover, the miR-101 mimic was able to reverse the inhibitory effect of the MALAT1-vector on expression of caspase-3 and caspase-8, while the miR-101 mimic attenuated the MALAT1-siRNA-induced facilitating effect on SCC9 cell apoptosis (Fig. 4C). A similar trend for the
Transwell assay is showed in Fig. 4D and E, overexpression of miR-101 inhibited the promotive role of the MALAT1-vector in SCC9 cell invasion, otherwise, miR-101 mimic was able to reverse the suppression of MALAT1-siRNA in regards to cell invasion. Collectively, these findings indicate that MALAT1 facilitates cell proliferation and invasion via suppressing miR-101 in OSCC cells.

EZH2 is a target gene of miR-101 in OSCC cells. TargetScan online software (http://www.targetscan.org/vert_71/) was used to detect the potential target of miR-101. It was found that EZH2 contains a putative binding site for miR-101 as shown in Fig. 5A. The results of dual-luciferase reporter assay indicated that the miR-101 mimic significantly reduced the luciferase activity of EZH2 3'-UTR-WT in the SCC9 cells. However, no significant change was noted in the EZH2 3'-UTR-MuT in SCC9 cells (Fig. 5B). Moreover, the result of qPCR indicated that $\mathrm{EZH} 2$ expression was significantly higher in OSCC tissues in comparison with that in the normal tissues (Fig. 5C). Overexpression of miR-101 suppressed EZH2 expression, while silencing of miR-101 expression enhanced EZH2 expression both at the mRNA and protein levels (Fig. 5D and E). These findings suggest that EZH2 is a direct target of miR-101.

EZH2 acts as a downstream gene of MALAT1 in OSCC cells. Then, we investigated whether EZH2 is regulated by MALAT1 through miR-101. As shown in Fig. 6A, EZH2 mRNA and protein expression was significantly enhanced by MALAT1 overexpression and then inhibited by miR-101 mimic. However, silencing of MALAT1 expression inhibited 


\section{A}
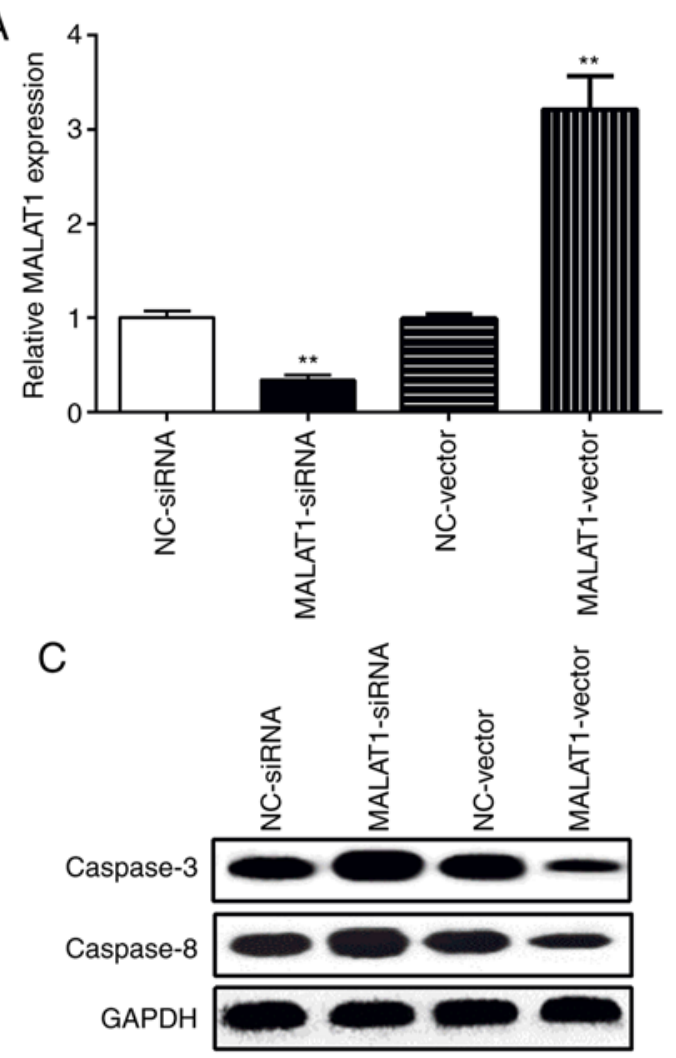

$\mathrm{D}$
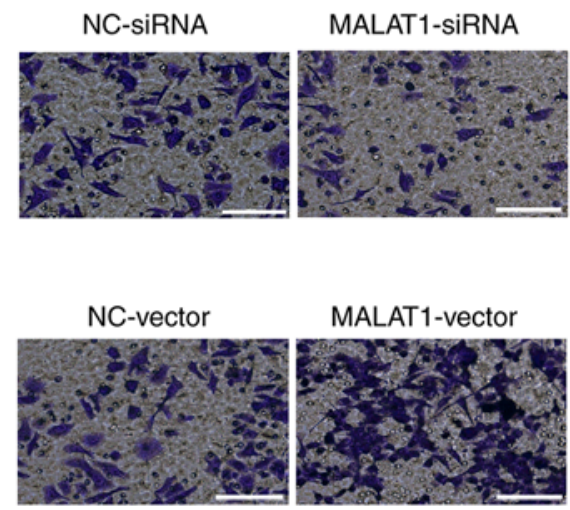

B
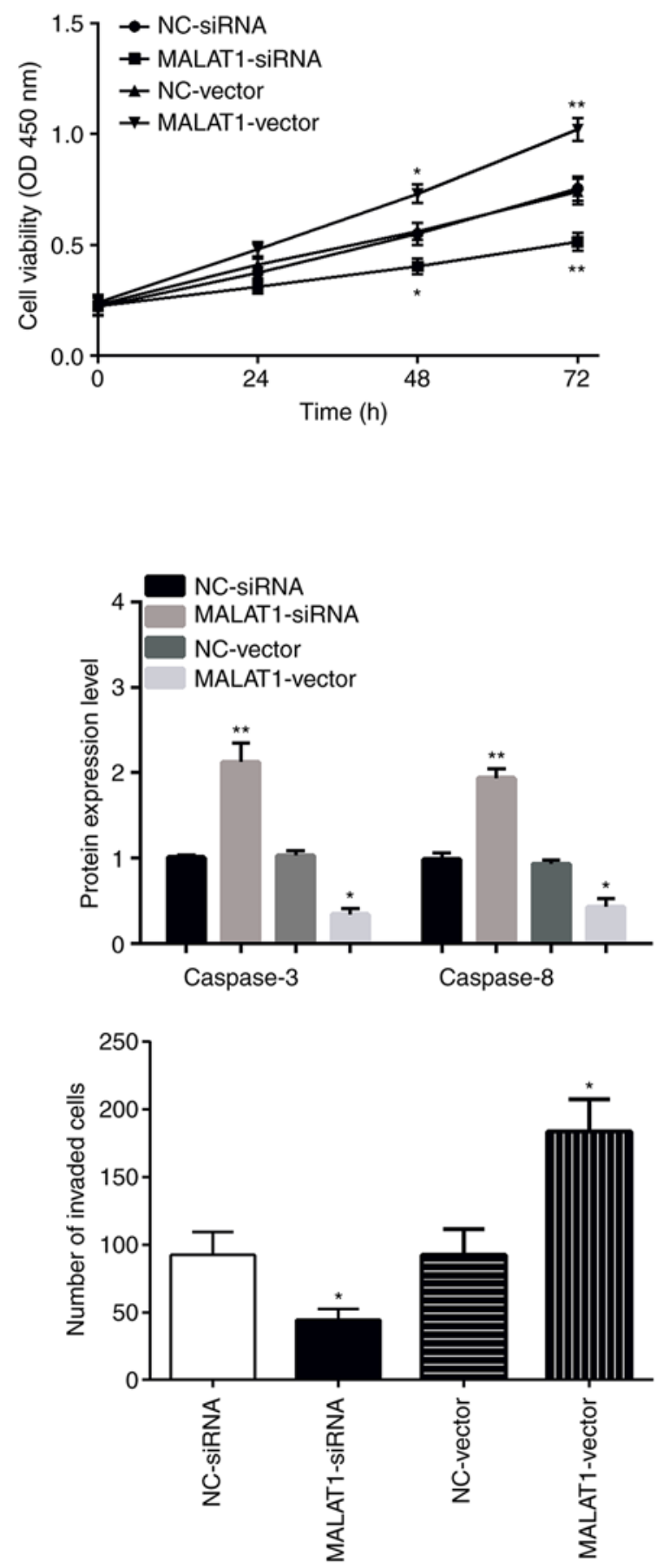

Figure 2. MALAT1 promotes OSCC cell viability and invasion. (A) qPCR analysis of the transfection efficiency in SCC9 cells after knockdown of MALAT1 (MALAT1-siRNA) or overexpression of MALAT1 (MALAT1-vector). (B) CCK-8 assy was used to detect the viability of the SCC9 cells following transfection. (C) Western blot analysis was used to detect the caspase-3 and caspase-8 levels in the SCC9 cells following transfection. (D) Transwell analysis was used to detect the invasion ability of the SCC 9 cells. ${ }^{*} \mathrm{P}<0.05,{ }^{* *} \mathrm{P}<0.01$ compared to the NC-siRNA or NC-vector group. OSCC, oral squamous cell carcinoma; MALAT1, metastasis associated lung adenocarcinoma transcript 1.

EZH2 expression while miR-101 mimic further suppressed EZH2 expression (Fig. 6B). In addition, the results of Pearson's correlation coefficient demonstrated that EZH2 expression was positively associated with MALAT1 expression (Fig. 6C), but negatively correlated with miR-101 expression in OSCC tissues (Fig. 6D). As shown in Fig. 6E, EZH2 overexpression facilitated SCC9 cell viability. The results of the western blot analysis displayed that the levels of caspase- 3 and caspase- 8 were decreased by the EZH2 plasmid (Fig. 6F). This may provide an added advantage to establish the oncogenic and tumor-suppressive roles of MALAT1 and miR-101 via EZH2.

\section{Discussion}

Long non-coding RNAs (lncRNAs) are a class of transcripts with more than 200 nucleotides that do not exhibit protein-coding abilities (17). Recent research has shown that IncRNAs play a pivotal role in the development of many 
A

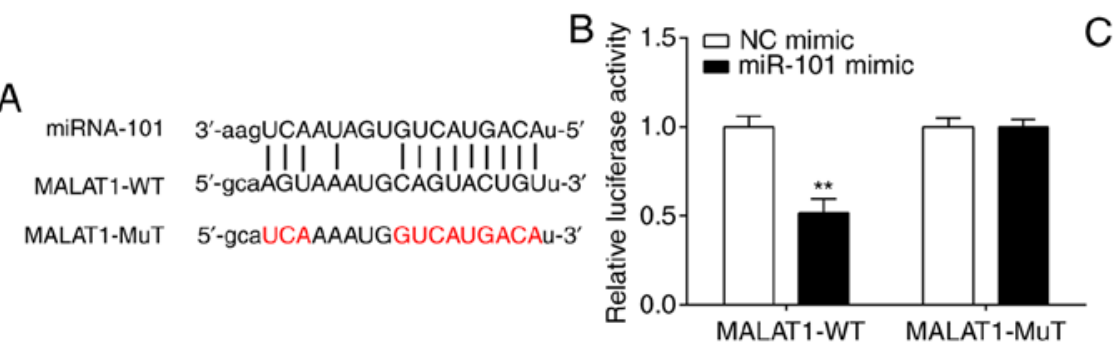

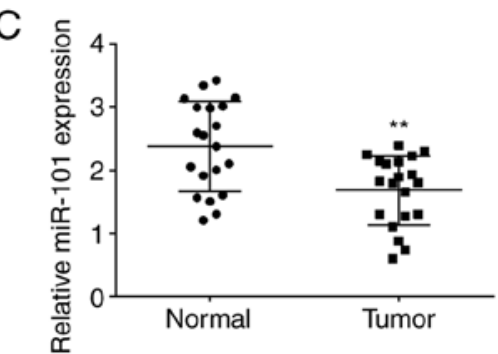

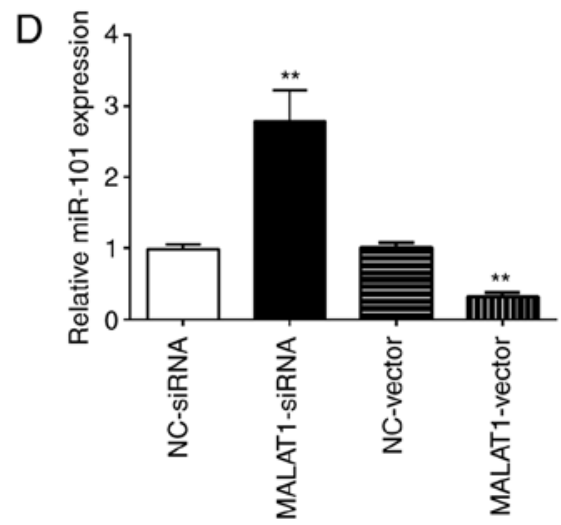

E

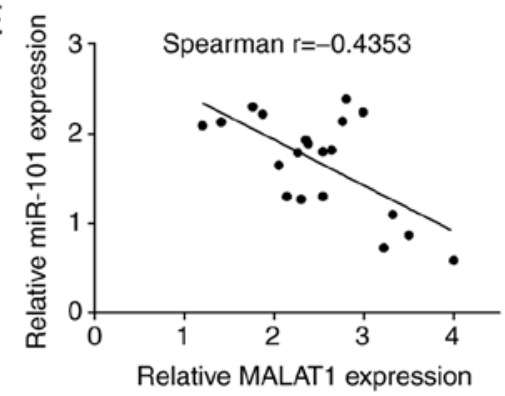

Figure 3. MALAT1 directly targets miR-101. (A) Starbase online software was used to predict the putative binding sites of miR-101 with MALAT1. (B) Luciferase activity of MALAT1 3'-UTR-WT (wild-type) or MuT (mutant) in SCC9 cells. ${ }^{\text {** }} \mathrm{P}<0.01$, compared with the NC-mimic group. (C) miR-101 expression in OSCC and normal tissues was detected by qPCR. ${ }^{* *} \mathrm{P}<0.01$, compared to the normal tissues. (D) qsPCR analysis was used to detect the miR-101 expression in SCC9 cells transfected with MALAT1-vector (overexpression) and MALAT1-siRNA (knockdown). ${ }^{* *} \mathrm{P}<0.01$, compared with the NC-siRNA or NC-vector group. (E) Correlation between MALAT1 and miR-101 in OSCC tissues was detected by Pearson's correlation coefficient. OSCC, oral squamous cell carcinoma; MALAT1, metastasis associated lung adenocarcinoma transcript 1.
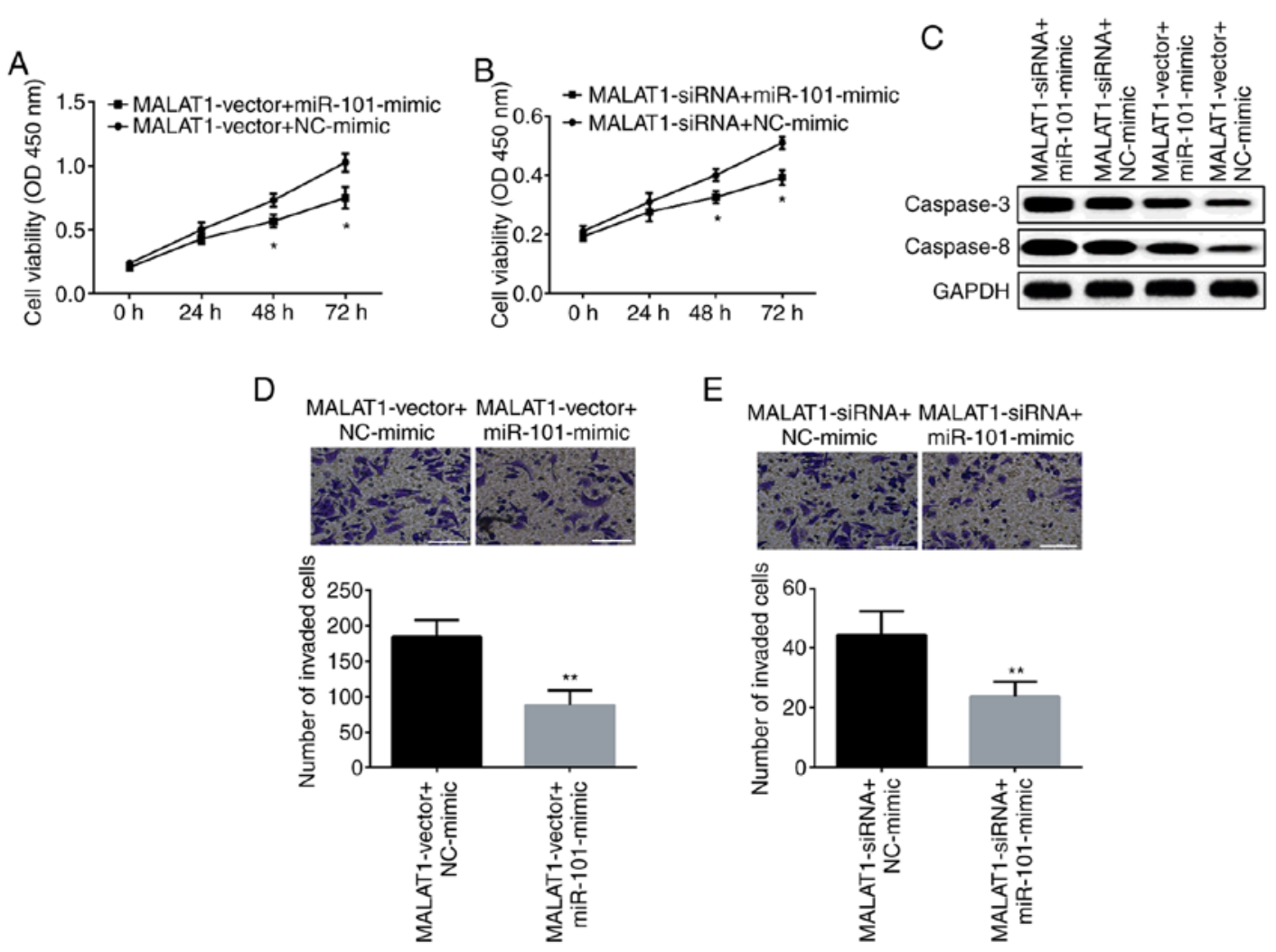

Figure 4. MALAT1 facilitates OSCC cell viability and invasion via modulating miR-101. (A and B) The viability of SCC9 cells following transfection was detected by CCK- 8 assay. "P $<0.05$, compared with the +miR-101-mimic transfected group. (C) Western blot analysis was used to detect the caspase-3 and caspase-8 levels in SCC9 cells in the different cell groups. (D and E) Transwell assay analysis was used to detect SCC9 cell invasion in the different cell groups. ${ }^{* *} \mathrm{P}<0.01$, compared with the +miR-101-mimic transfected group. OSCC, oral squamous cell carcinoma; MALAT1, metastasis associated lung adenocarcinoma transcript 1 . 

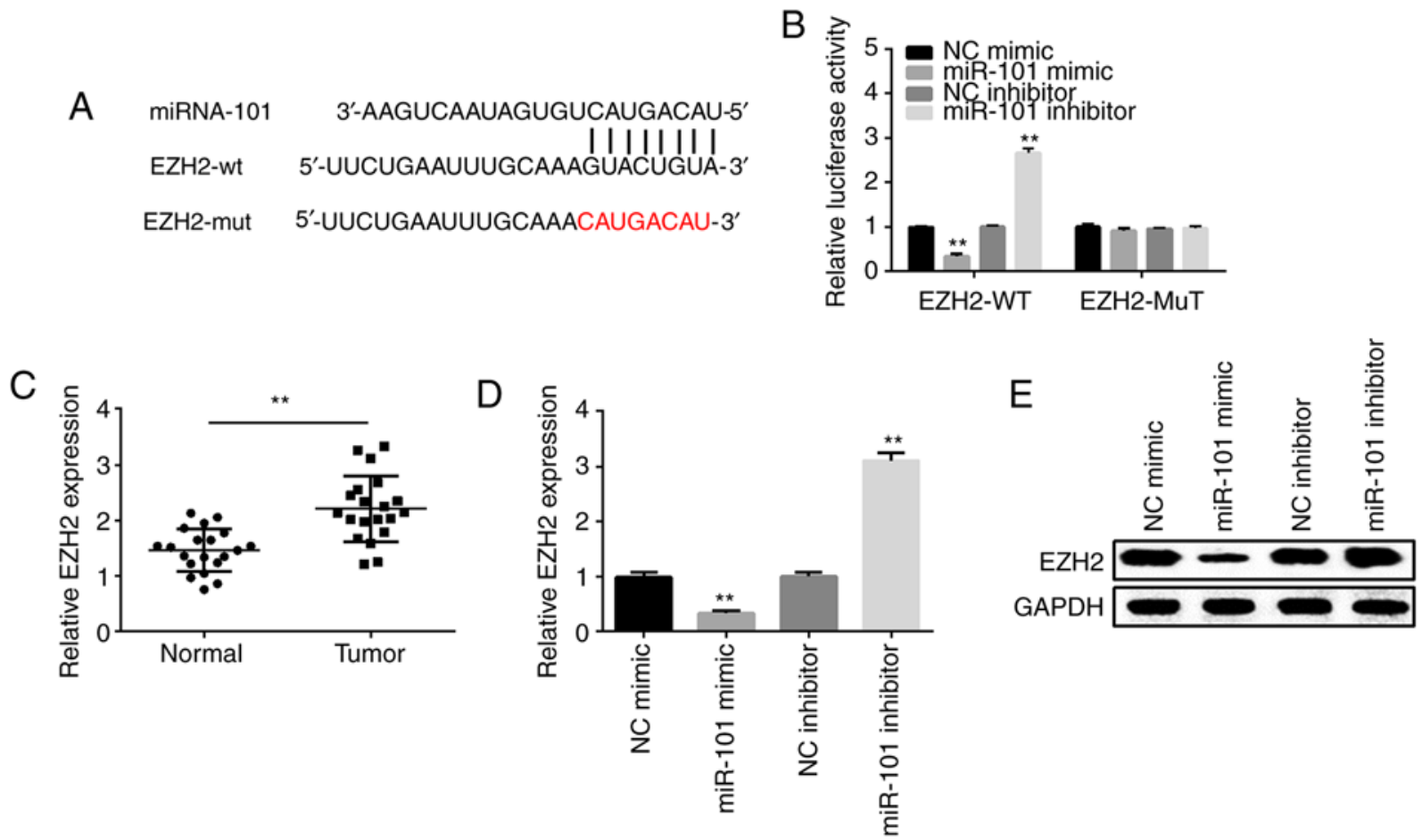

Figure 5. EZH2 is a target of miR-101. (A) Prediction the putative binding sites of miR-101 with EZH2. (B) Luciferase activity of EZH2 3'-UTR-WT (wild-type) or MuT (mutant) in SCC9 cells. ${ }^{* *} \mathrm{P}<0.01$, compared with the NC mimic or NC inhibitor group. (C) Measurement of EZH2 expression in OSCC and normal tissues by qPCR. ${ }^{* *} \mathrm{P}<0.01$. (D) qPCR and (E) western blot analysis were used to detect EZH2 expression in the SCC9 cell groups. ${ }^{* *} \mathrm{P}<0.01$, compared with the $\mathrm{NC}$ mimic or NC inhibitor group. OSCC, oral squamous cell carcinoma; EZH2, Enhancer of zeste 2 polycomb repressive complex 2 subunit.

tumors, but the related mechanism remains to be investigated (18). In this study, we revealed that metastasis associated lung adenocarcinoma transcript 1 (MALAT1) was upregulated in oral squamous cell carcinoma (OSCC) tissues and cell lines (HSC3, SCC9, SCC15 and SCC25).

MALAT1 is an important lncRNA in tumor progression. Previous studies have shown that MALAT1 is upregulated in colorectal cancer (CRC), pancreatic ductal adenocarcinoma and head and neck squamous cell carcinoma (19-21). Moreover, Xu et al displayed that MALAT1 overexpression promoted the viability and migration of CRC (22). Pan et al found that overexpression of MALAT1 enhanced the invasion and migration of hepatocellular carcinoma (23). Huang et al implicated the role of MALAT1 in facilitating the angiogenesis of breast cancer (24). Zhu et al found that MALAT1 displayed facilitating effect on gastric cancer cell proliferation and metastasis (25). Moreover, Lin et al displayed that MALAT1 was upregulated in gallbladder cancer and its high expression was associated with poor prognosis (26). Stone et al showed high expression of MALAT1 in breast cancer and MALAT1 was associated with tumor development (27). Moreover, Zhou et al revealed that MALAT1 was elevated in OSCC and promoted tumor growth and metastasis (28). Our findings revealed that MALAT1 enhanced OSCC cell proliferation and invasion, which supporting previous observations that MALAT1 acts as an oncogene in OSCC development (29). The results of this study also displayed that the levels of caspase- 3 and caspase- 8 were decreased by MALAT1 overexpression, while increased by silencing of MALAT1. In the present study, we chose to investigate caspase- 3 and caspase- 8 but not caspase- 9 , which may be the limitation of the present study.
As the development of RNA-sequencing technologies, many non-coding RNAs have been identified. IncRNAs possess important functions in development, immunology and cancer. For example, lncRNA CASC2 was found to be downregulated in OSCC and markedly inhibited cell proliferation via targeting microRNA-21 (30). On the other hand, Zhang et al showed that IncRNA PAPAS conferred a promotive effect on OSCC development as an oncogene by modulating the TGF- $\beta 1$ signaling pathway (31). In addition, lncRNA LEF1-AS1 was found to act as an oncogene in OSCC progression and silencing of LEF1-AS1 suppressed cell proliferation, migration and tumor growth through the Hippo signaling pathway (32).

Recently, a growing number of reports have suggested that IncRNAs act as 'sponges' to bind specific miRNAs and regulate their function. Previous research has established that MALAT1 promoted hepatocellular carcinoma cell migration and invasion by targeting miR-142-3p (33). Chang and Hu identified the biological function of MALAT1 in promoting OSCC development via targeting the miR-125b/STAT3 axis (29). Here, we showed that MALAT1 is a target of miR-101 by bioinformatics analysis and luciferase reporter assays. Thus, our research is the first to report that MALAT1 regulates OSCC proliferation and invasion by targeting miR-101.

By using TargetScan, we predicted Enhancer of zeste 2 polycomb repressive complex 2 subunit (EZH2) as a direct target of miR-101. Moreover, luciferase reporter assay further determined EZH2 as a direct target of miR-101. EZH2 has been reported to be involved in OSCC progression as the target of several miRNAs, such as miR-200, miR-138 and miR-32 (34-36). Here, we demonstrated that MALAT1 can 

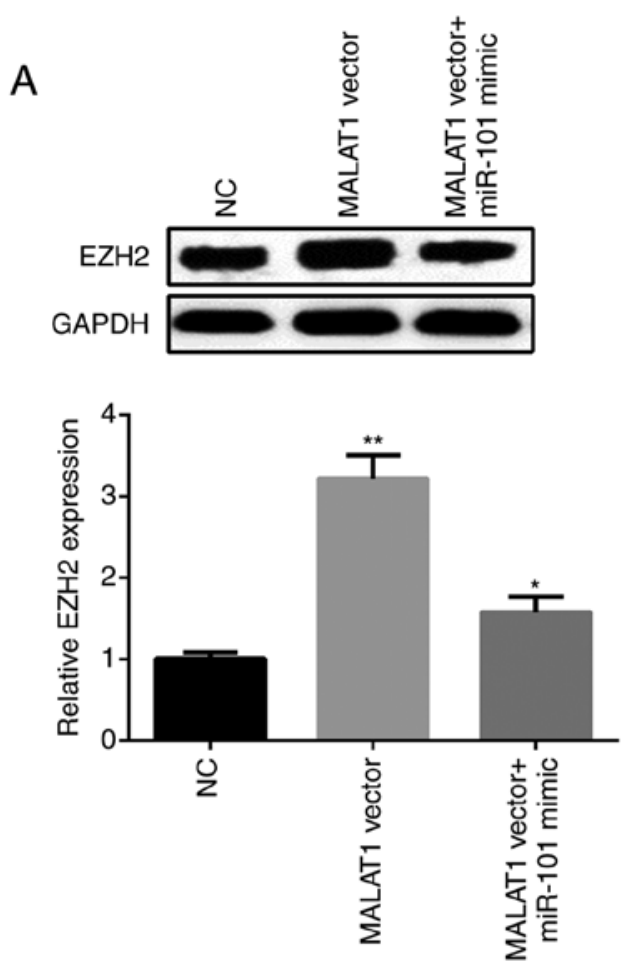

C

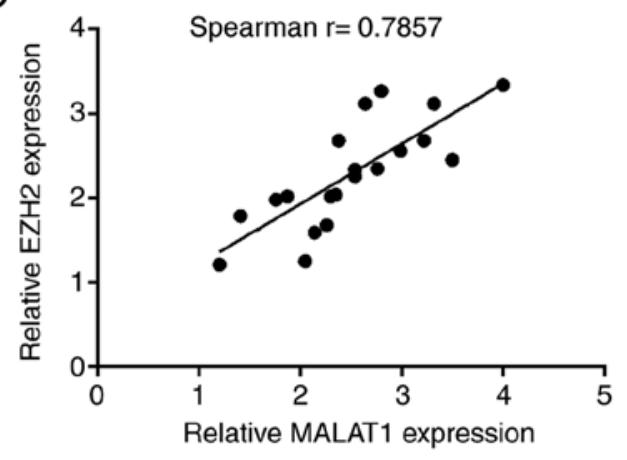

$\mathrm{E}$

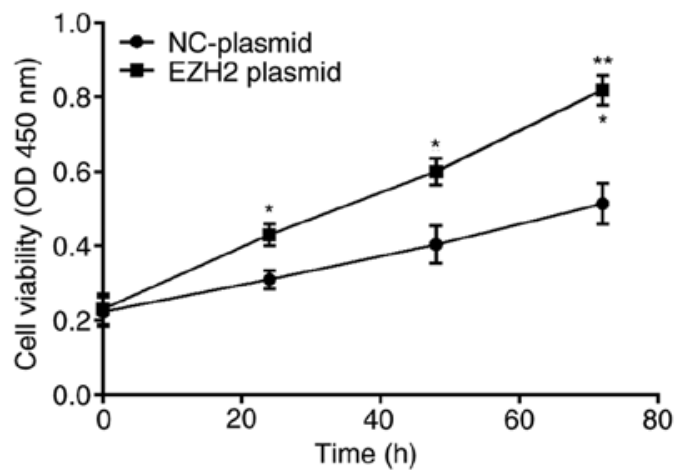

B
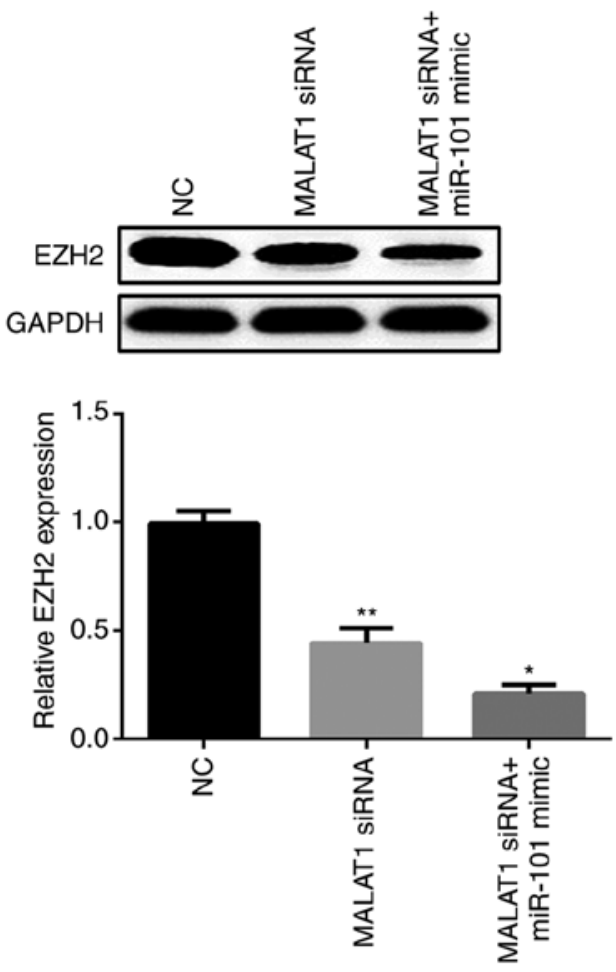

D

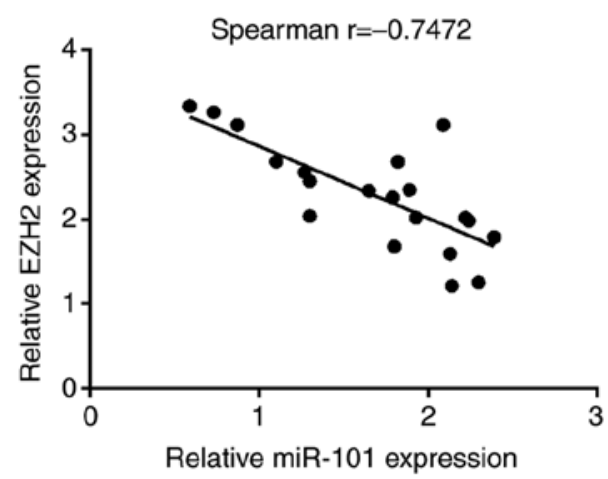

$\mathrm{F}$

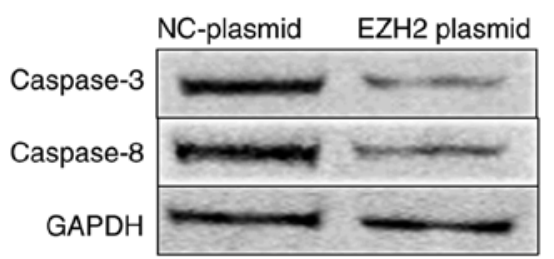

Figure 6. EZH2 acts as a downstream effecter of MALAT1 in OSCC cells. (A and B) EZH2 mRNA and protein expression were detected by qPCR and western blot analysis in SCC9 cells following transfection with the MALAT-vector with or without the miR-101 mimic. ${ }^{*} \mathrm{P}<0.05,{ }^{* *} \mathrm{P}<0.01$, compared with the NC group. (C) Positive correlation of EZH2 and MALAT1 expression was detected by Pearson's correlation coefficient. (D) Negative correlation of EZH2 and miR-101 expression was detected by Pearson's correlation coefficient. (E) EZH2 overexpression facilitated SCC9 cell viability. ${ }^{*} \mathrm{P}<0.05,{ }^{* *} \mathrm{P}<0.01$, compared with the NC-Plasmid group. (F) Results of western blot analysis displayed that the levels of caspase-3 and caspase- 8 were decreased by the EZH2-plasmid. OSCC, oral squamous cell carcinoma; MALAT1, metastasis associated lung adenocarcinoma transcript 1; EZH2, Enhancer of zeste 2 polycomb repressive complex 2 subunit.

regulate EZH2 expression by modulating miR-101 expression. Overexpression of MALAT1 significantly promoted EZH2 expression, while treatment with the miR-101 mimic attenuated EZH2 expression. Conversely, knockdown of MALAT1 inhibited EZH2 expression, while transfection with the miR-101 mimic further suppressed EZH2 expression. 
In conclusion, the present study aimed to discover the biological function and molecular mechanism of lncRNA MALAT1 in OSCC development. The findings showed that MALAT1 was upregulated, while miR-101 was downregulated in OSCC tissues and cell lines. MALAT1 modulated OSCC progression by negatively regulating miR-101. Moreover, EZH2 was determined as the target of miR-101 by bioinformatics analysis. Taken together, MALAT1 regulates EZH2 expression by modulating miR-101, which may be considered as a novel target in OSCC treatment.

\section{Acknowledgements}

Not applicable.

\section{Funding}

Not applicable.

\section{Availability of data and materials}

The datasets used and/or analyzed during the present study are available from the corresponding author on reasonable request.

\section{Authors' contributions}

LX first author contributed significantly to analysis and manuscript preparation. WW as the co-first author performed the data analyses and wrote the manuscript. HX as the corresponding author contributed to the conception of the study. JZ, SL and XY helped perform the analyses with constructive discussions. All authors read and approved the manuscript and agree to be accountable for all aspects of the research in ensuring that the accuracy or integrity of any part of the work are appropriately investigated and resolved.

\section{Ethics approval and consent to participate}

The study was approved by the Ethics Committee of the Affiliated Yantai Yuhuangding Hospital of Qingdao University. Signed written informed consents were obtained from the patients and/or guardians.

\section{Patient consent for publication}

Not applicable.

\section{Competing interests}

The authors declare that they have no competing interests.

\section{References}

1. Choi S and Myers JN: Molecular pathogenesis of oral squamous cell carcinoma: Implications for therapy. J Dent Res 87: 14-32, 2008.

2. Johnson NW, Jayasekara P and Amarasinghe AA: Squamous cell carcinoma and precursor lesions of the oral cavity: Epidemiology and aetiology. Periodontol 2000 57: 19-37, 2011.

3. Ferlay J, Soerjomataram I, Dikshit R, Eser S, Mathers C, Rebelo M, Parkin DM, Forman D and Bray F: Cancer incidence and mortality worldwide: Sources, methods and major patterns in GLOBOCAN 2012. Int J Cancer 136: E359-E386, 2015.
4. Sinevici N and O'sullivan J: Oral cancer: Deregulated molecular events and their use as biomarkers. Oral Oncol 61: 12-18, 2016.

5. Wang S, Hui Y, Li X and Jia Q: Silencing of lncRNA CCDC26 restrains the growth and migration of glioma cells in vitro and in vivo via targeting miR-203. Oncol Res 26: 1143-1154, 2018.

6. Zhang JJ, Wang DD, Du CX and Wang Y: Long noncoding RNA ANRIL promotes cervical cancer development by acting as a sponge of miR-186. Oncol Res 26: 345-352, 2018.

7. Gao YL, Zhao ZS, Zhang MY, Han LJ, Dong YJ and Xu B: Long noncoding RNA PVT1 facilitates cervical cancer progression via negative regulating of miR-424. Oncol Res 25: 1391-1398, 2017.

8. Hua F, Li CH, Chen XG and Liu XP: Long noncoding RNA CCAT2 knockdown suppresses tumorous progression by sponging miR-424 in epithelial ovarian cancer. Oncol Res 26: 241-247, 2018.

9. Ji P, Diederichs S, Wang W, Böing S, Metzger R, Schneider PM, Tidow N, Brandt B, Buerger H, Bulk E, et al: MALAT-1, a novel noncoding RNA, and thymosin beta4 predict metastasis and survival in early-stage non-small cell lung cancer. Oncogene 22: 8031-8041, 2003

10. Zheng HT, Shi DB, Wang YW, Li XX, Xu Y, Tripathi P, Gu WL, Cai GX and Cai SJ: High expression of lncRNA MALAT1 suggests a biomarker of poor prognosis in colorectal cancer. Int J Clin Exp Pathol 7: 3174-3181, 2014.

11. Croce CM: Causes and consequences of microRNA dysregulation in cancer. Nat Rev Genet 10: 704-714, 2009.

12. Wu B, Lei D, Wang L, Yang X, Jia S, Yang Z, Shan C, Yang X, Zhang C and Lu B: MiRNA-101 inhibits oral squamous-cell carcinoma growth and metastasis by targeting zinc finger E-box binding homeobox 1. Am J Cancer Res 6: 1396-1407, 2016.

13. Jiang M, Xu B, Li X, Shang Y, Chu Y, Wang W, Chen D, Wu N, $\mathrm{Hu}$ S, Zhang S, et al: O-GlcNAcylation promotes colorectal cancer metastasis via the miR-101-O-GlcNAc/EZH2 regulatory feedback circuit. Oncogene 38: 301-316, 2019.

14. Chen L, Jia J, Zang Y, Li J and Wan B: MicroRNA-101 regulates autophagy, proliferation and apoptosis via targeting EZH2 in laryngeal squamous cell carcinoma. Neoplasma 66: 507-515, 2019.

15. Jin Q, He W, Chen L, Yang Y, Shi K and You Z: MicroRNA-101-3p inhibits proliferation in retinoblastoma cells by targeting EZH2 and HDAC9. Exp Ther Med 16: 1663-1670, 2018.

16. Livak KJ and Schmittgen TD: Analysis of relative gene expression data using real-time quantitative PCR and the 2(-Delta Delta C(T)) method. Methods 25: 402-408, 2001.

17. Bertone P, Stolc V, Royce TE, Rozowsky JS, Urban AE, Zhu X Rinn JL, Tongprasit W, Samanta M, Weissman S, et al: Global identification of human transcribed sequences with genome tiling arrays. Science 306: 2242-2246, 2004.

18. Zhang H, Chen Z, Wang X, Huang Z, He Z and Chen Y: Long non-coding RNA: A new player in cancer. J Hematol Oncol 6: 37, 2013.

19. Tang D, Yang Z, Long F, Luo L, Yang B, Zhu R, Sang X and Cao G: Inhibition of MALAT1 reduces tumor growth and metastasis and promotes drug sensitivity in colorectal cancer. Cell Signal 57: 21-28, 2019.

20. Yao Y, Chen X, Lu S, Zhou C, Xu G, Yan Z, Yang J, Yu T, Chen W, Qian Y, et al: Circulating long noncoding RNAs as biomarkers for predicting head and neck squamous cell carcinoma. Cell Physiol Biochem 50: 1429-1440, 2018.

21. Zhuo M, Yuan C, Han T, Cui J, Jiao F and Wang L: A novel feedback loop between high MALAT-1 and low miR-200c-3p promotes cell migration and invasion in pancreatic ductal adenocarcinoma and is predictive of poor prognosis. BMC Cancer 18: $1032,2018$.

22. Xu Y, Zhang X, Hu X, Zhou W, Zhang P, Zhang J, Yang S and Liu Y: The effects of lncRNA MALAT1 on proliferation, invasion and migration in colorectal cancer through regulating SOX9. Mol Med 24: 52, 2018

23. Pan Y, Tong S, Cui R, Fan J, Liu C, Lin Y, Tang J, Xie H, Lin P, Zheng $\mathrm{T}$ and $\mathrm{Yu} \mathrm{X}$ : Long non-coding MALAT1 functions as a competing endogenous RNA to regulate vimentin expression by sponging miR-30a-5p in hepatocellular carcinoma. Cell Physiol Biochem 50: 108-120, 2018.

24. Huang XJ, Xia Y, He GF, Zheng LL, Cai YP, Yin Y and Wu Q: MALAT1 promotes angiogenesis of breast cancer. Oncol Rep 40: 2683-2689, 2018

25. Zhu K, Ren Q and Zhao Y: lncRNA MALAT1 overexpression promotes proliferation, migration and invasion of gastric cancer by activating the PI3K/AKT pathway. Oncol Lett 17: 5335-5342, 2019. 
26. Lin N, Yao Z, Xu M, Chen J, Lu Y, Yuan L, Zhou S, Zou X and Xu R: Long noncoding RNA MALAT1 potentiates growth and inhibits senescence by antagonizing ABI3BP in gallbladder cancer cells. J Exp Clin Cancer Res 38: 244, 2019.

27. Stone JK, Kim JH, Vukadin L, Richard A, Giannini HK, Lim SS, Tan M and Ahn EE: Hypoxia induces cancer cell-specific chromatin interactions and increases MALAT1 expression in breast cancer cells. J Biol Chem 294: 11213-11224, 2019.

28. Zhou X, Liu S, Cai G, Kong L, Zhang T, Ren Y, Wu Y, Mei M, Zhang L and Wang X: Long Non-coding RNA MALAT1 promotes tumor growth and metastasis by inducing epithelial-mesenchymal transition in oral squamous cell carcinoma. Sci Rep 5: 15972, 2015.

29. Chang SM and Hu WW: Long non-coding RNA MALAT1 promotes oral squamous cell carcinoma development via microRNA-125b/STAT3 axis. J Cell Physiol 233: 3384-3396, 2018.

30. Pan L, Chen H, Bai Y, Wang Q and Chen L: Long non-coding RNA CASC2 serves as a ceRNA of microRNA-21 to promote PDCD4 expression in oral squamous cell carcinoma. Onco Targets Ther 12: 3377-3385, 2019.

31. Zhang P, Liu Y, Li C, Zhang L, Liu Q and Jiang T: LncRNA PAPAS promotes oral squamous cell carcinoma by upregulating transforming growth factor- $\beta 1$. J Cell Biochem 120: 16120-16127, 2019.
32. Zhang C, Bao C, Zhang X, Lin X, Pan D and Chen Y: Knockdown of lncRNA LEF1-AS1 inhibited the progression of oral squamous cell carcinoma (OSCC) via Hippo signaling pathway. Cancer Biol Ther 20: 1213-1222, 2019.

33. Yu Q, Xiang L, Chen Z, Liu X, Ou H, Zhou J and Yang D MALAT1 functions as a competing endogenous RNA to regulate SMAD5 expression by acting as a sponge for miR-142-3p in hepatocellular carcinoma. Cell Biosci 9: 39, 2019.

34. Wang Y, Guo W, Li Z, Wu Y, Jing C, Ren Y, Zhao M, Kong L, Zhang C, Dong J, et al: Role of the EZH2/miR-200 axis in STAT3-mediated OSCC invasion. Int J Oncol 52: 1149-1164, 2018.

35. Hong Y, He H, Sui W, Zhang J, Zhang S and Yang D: Long non-coding RNA H1 promotes cell proliferation and invasion by acting as a ceRNA of miR-138 and releasing EZH2 in oral squamous cell carcinoma. Int J Oncol 52: 901-912, 2018.

36. Zhang D, Ni Z, Xu X and Xiao J: miR-32 functions as a tumor suppressor and directly targets EZH2 in human oral squamous cell carcinoma. Med Sci Monit 20: 2527-2535, 2014.

(i) $(9$ This work is licensed under a Creative Commons Attribution-NonCommercial-NoDerivatives 4.0 International (CC BY-NC-ND 4.0) License. 